\title{
ORBITAL FLOOR STEROID INJECTIONS IN THE TREATMENT OF UVEITIS
}

\author{
P. RIORDAN-EVA and S. LIGHTMAN \\ London
}

\begin{abstract}
SUMMARY
Orbital floor steroid injections, like other posterior periocular injections, are advocated as a means of avoiding systemic immunosuppression in the management of uveitis in which there is significant posterior segment inflammation, but there are no published studies on their efficacy. The results of 54 orbital floor steroid injections to 33 eyes with uveitis were analysed. A positive response with an average duration of effect of 9 weeks was obtained after $48 \%$ of injections. These results compare favourably with the results of systemic immunosuppression in patients with comparable patterns of uveitis, thus establishing the efficacy of orbital floor steroid injections and their potential value when systemic immunosuppression needs to be avoided.
\end{abstract}

Posterior segment activity in uveitis often requires systemic immunosuppression; however, this is associated with many side effects, including potentially life-threatening complications, and is better avoided if there is only mild intraocular inflammation, disease activity is confined to one eye, or there are specific contraindications such as pregnancy. The widely recommended alternative therapy is periocular steroid injections, either to the orbital floor or to the posterior sub-Tenon's space, which achieve high, potentially long-lasting local concentrations of active agent with less risk of systemic side effects. There is still the potential for local side effects, including globe perforation, but this is less likely with orbital floor than with posterior sub-Tenon's injections. Despite the theoretical advantages of orbital floor steroid injections in the treatment of posterior segment inflammation in uveitis, there are no previously published studies specifically assessing their efficacy.

\section{MATERIALS AND METHODS}

All the patients seen in the Uveitis Clinic at Moorfields

From: Uveitis Clinic, Moorfields Eye Hospital, London, UK

Correspondence to: $\mathrm{Mr} \mathrm{P}$. Riordan-Eva, FRCS, FRCOphth, Department of Neuro-Ophthalmology, National Hospital for Neurology and Neurosurgery, Queen Square, London WC1N 3BG, UK.
Eye Hospital during a 4 month period (1 December 1991 to 31 March 1992) who were treated during that time or had previously been treated at Moorfields by orbital floor steroid injection were reviewed. Details of the therapy and its effects, as well as the diagnosis, were obtained from the hospital notes. Patients were interviewed and examined by one of the authors (P.R.E.) to confirm these details.

Visual acuities were recorded as Snellen equivalents at a distance of $6 \mathrm{~m}$. Vitreous inflammatory activity was scored according to the recorded level of cellular activity $(+/-=1,+=2,++=3,+++=4)$. The degree of vitreous opacification or structural change within the vitreous was not used for analysis. Anterior chamber inflammatory activity was not analysed because of the influence of changes in concurrent topical steroid therapy. The presence or absence of macular or disc oedema on clinical examination was recorded for the purposes of sub-grouping the treated eyes, but in the absence of routine fluorescein angiography was not used to assess disease activity or response to treatment.

The criteria for therapeutic success were an improvement in visual acuity of 2 Snellen lines or more or an improvement of 2 or more in the vitreous cellular activity score. Duration of a positive response was determined by the timing of the last review examination at which improvement was still noted to be present.

Statistical analysis was performed with the chi-squared test for numerical data and with the Wilcoxon rank-sum test for ordinal and nominal data.

\section{RESULTS}

Twenty-eight patients were identified ( 16 female, 12 male; aged 15-75 (mean 39) years). A total of 54 injections (Depo-Medrone (Upjohn) 20-40 mg, methylprednisolone $40 \mathrm{mg}$ or triamcinolone $40 \mathrm{mg}$ ) had been administered to 33 eyes ( 22 right, 11 left) over a greater than 10 year period from 9 September 1981 to 19 February 1992. All injections had been administered through the eyelid. The injections had been administered by Consultants, Fellows, or Senior Registrars attached to the Uveitis Clinic. 
Because of the number of individuals involved it was not statistically valid to analyse the results according to who had administered the injection.

The commonest diagnoses were pars planitis and idiopathic uveitis (Table I). The indications for treatment were either a visual acuity of $6 / 12$ or less or symptoms related to vitreous cellular activity. At the time of 52 injections, acute disease activity was confined to the eye being treated. One patient received simultaneous injections to both eyes because of inflammatory activity in both eyes, there having been no response to a previous trial of systemic steroids but a beneficial response to a previous unilateral orbital floor steroid injection. Besides this consideration the prime reasons for selecting orbital floor steroid injection were:

1. Unilateral disease in patients not previously treated except with topical therapy.

2. Additional therapy in patients with unilateral exacerbation of disease while being successfully treated for bilateral disease with systemic steroids.

3. Unilateral disease relapse in patients who had previously responded to orbital floor steroid injection.

The total duration of disease activity in the treated eye at the time of injection ranged from 1 to 156 months (mean 29 months). Pre-treatment visual acuities ranged from $6 / 6$ to counting fingers (Fig. 1), with a median of $6 / 24$. Seven eyes had a pre-treatment acuity of $6 / 9$ or $6 / 6$. The pretreatment vitreous cellular activity score ranged from 0 to 4 with a mean of 2.1 (Fig. 2). None of the 7 eyes with an acuity of better than $6 / 12$ had a vitreous cellular activity score of less than 2 before treatment.

It would be expected that the pattern of response in individual eyes and between the two eyes of the same patient would be interdependent and that the responses could not be analysed independently. Surprisingly this was not the case. A total of 33 injections were administered to the 11 eyes that underwent repeated injections. Amongst these 33 injections the response to one particular injection could not be predicted by the response to previous or to subsequent injections to the same eye, i.e. a positive response to one injection was just as likely to be followed by a negative or a positive response to the next injection to the same eye. Thus first and repeat injections to the same eye had the same likelihood of producing a positive response and an initial response was not associated with an increased likelihood of a positive response to subsequent injections. Similarly amongst 5 patients who underwent treatment to

Table I. Diagnoses

\begin{tabular}{lcc}
\hline & Patients & Eyes \\
\hline Pars planitis & 11 & 13 \\
Idiopathic uveitis & 10 & 13 \\
Idiopathic retinal vasculitis & 2 & 2 \\
Sarcoidosis & 2 & 2 \\
Multifocal choroiditis & 1 & 1 \\
Sympathetic uveitis & 1 & 1 \\
Herpes zoster & 1 & 1 \\
Total & 28 & 33 \\
\hline
\end{tabular}

both eyes, 4 had a different response to the first injection administerd to each of the two eyes, e.g. a positive response in one eye and a negative response in the fellow eye, and only 1 patient had the same response in the two eyes. Thus amongst the factors controlling the response to orbital floor steroid injections the individual patient's or individual eye's response to previous treatment was not significant. For this reason and the simplification that ensues, the results are reported and discussed with respect to individual eyes. The details of response rates and mean duration of response according to various parameters are given in Table II.

There was a positive response to $48 \%$ of injections with a duration of effect ranging from 2 to 26 weeks (mean 9 weeks). Twenty of the 54 injections $(37 \%)$ produced a positive response for 4 weeks or more. Nineteen injections ( $40 \%$ of those administered to eyes with a pre-treatment acuity worse than 6/9) produced an improvement in visual acuity, with a range of 2 to 5 Snellen lines and a mean improvement of 2.7 lines (Fig. 1). Thirteen of these 19 injections did not result in a coincidental improvement in cellular activity. In these 13 cases the eyes had a lower mean vitreous cellular activity score ( 1.6 vs. $2.7, p<0.05)$ but the same prevalence of macular oedema (69\% vs. $67 \%$ ) before treatment compared with the eyes treated with the six injections which produced an improvement in both visual acuity and inflammatory activity. There was no difference in the degree of visual acuity improvement between the groups with and without improvement in cellular activity. Post-treatment vitreous cellular activity scores were not available for 3 injections. Of the other 51 injections, 13 produced an improvement in the vitreous cellular activity score, with a range from 2 to 5 and a mean improvement of 2.5 (Fig. 2).

There was no statistical difference in treatment outcome according to sex or age of the patients, disease duration, visual acuity, the presence of macular or disc oedema prior to treatment, or the type or dose of injection used. Also there was no difference in outcome between eyes with pars planitis and those with idiopathic uveitis. Of the 7 injections administered to eyes with a pre-treatment visual acuity of $6 / 9$ or $6 / 6$ there was a positive response to $4(57 \%)$. Seven of 9 eyes that did not respond to orbital floor steroid injection and were treated with systemic steroids improved with the latter treatment. Conversely 5 of 6 eyes which had failed to respond to systemic steroid therapy and were treated with orbital floor steroids responded to the latter treatment.

Five eyes suffered a 1 line decrease in visual acuity, 4 eyes suffered an increase in the cellular activity score of 1 , and 1 eye suffered an increase in cellular activity score of 2. No eyes suffered a drop in visual acuity of 2 or more lines or an increase of 3 or more in the vitreous cellular activity score. Thus only 1 eye showed an adverse response after orbital floor steroid injection using the level of criteria set for a positive response. There were no complications from the orbital floor steroid injections in this group. 


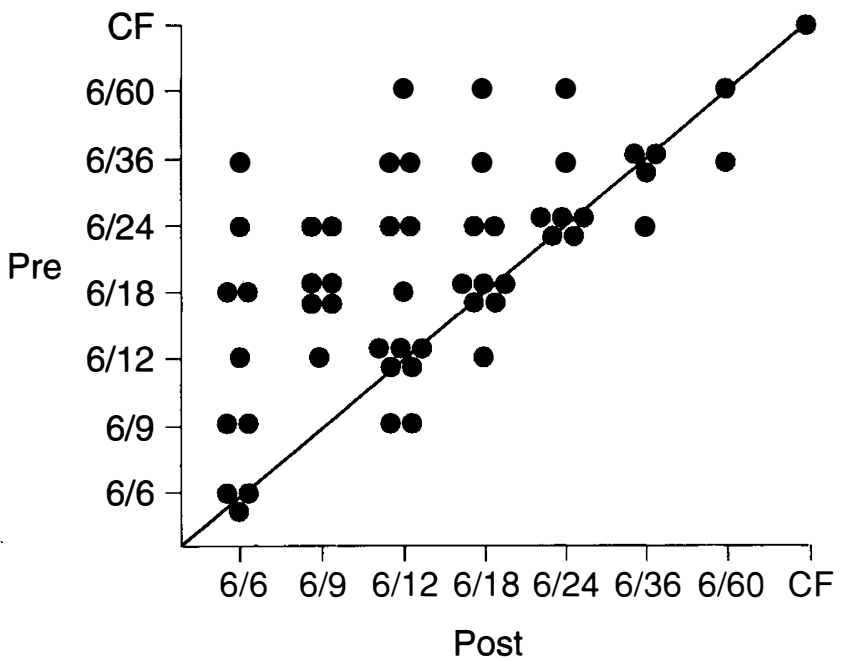

Fig. 1. Pre- and post-treatment visual acuities (54 injections).

\section{DISCUSSION}

Experimental studies have clearly demonstrated that posterior periocular steroid injections produce high concentrations of active agent within the posterior segment of the eye, producing higher levels than systemic steroids particularly when the eye is inflamed. ${ }^{1,2}$ This combined with the low risk of systemic side effects has led to a widespread promulgation of the advantages of posterior periocular steroid injections in the management of uveitis. ${ }^{3.4}$ However there is very little published information on their efficacy. In a general review of periocular steroid injections for ocular inflammatory disease, Nozik ${ }^{5}$ reported on the use of 25 posterior sub-Tenon's and 18 retrobulbar injections in the treatment of uveitis. Unfortunately the outcome of these injections is not specifically recorded. The overall success rate including the results of 138 subconjunctival or anterior sub-Tenon's injections was that $86 \%$ of the patients treated benefited, the majority receiving more than two injections. Godfrey et al. ${ }^{6}$ reported sustained improvement in $71 \%$ of eyes with pars planitis treated with repeated periocular steroid injections, without

Table II. Results of orbital floor steroid injections according to individual eyes

\begin{tabular}{lcccc}
\hline & Eyes & Injections & $\begin{array}{c}\text { Positive } \\
\text { response } \\
(\%)\end{array}$ & $\begin{array}{c}\text { Average } \\
\text { duration } \\
\text { (weeks) }\end{array}$ \\
\hline All injections & 33 & 54 & 48 & 9 \\
$\begin{array}{l}\text { Idiopathic uveitis } \\
\text { Pars planitis }\end{array}$ & 13 & 19 & 53 & 6 \\
$\begin{array}{l}\text { Pre-treatment visual acuity } \\
\text { 6/9 or better }\end{array}$ & 24 & 46 & 10 \\
$\quad$ 6/18 or better & 7 & 57 & 9 \\
$\quad$ 6/24 or worse & 27 & 44 & 10 \\
$\begin{array}{l}\text { Disease duration prior to treatment } \\
\text { Less than 6 months }\end{array}$ & 27 & 52 & 8 \\
$\quad$ 6 months or greater & & 38 & 56 & 11 \\
Pre-treatment macular & 21 & 36 & 44 & 7 \\
$\quad$ oedema & 5 & 5 & 80 & 7 \\
$\begin{array}{l}\text { Pre-treatment } \\
\text { disc oedema }\end{array}$ & & & & \\
\hline
\end{tabular}

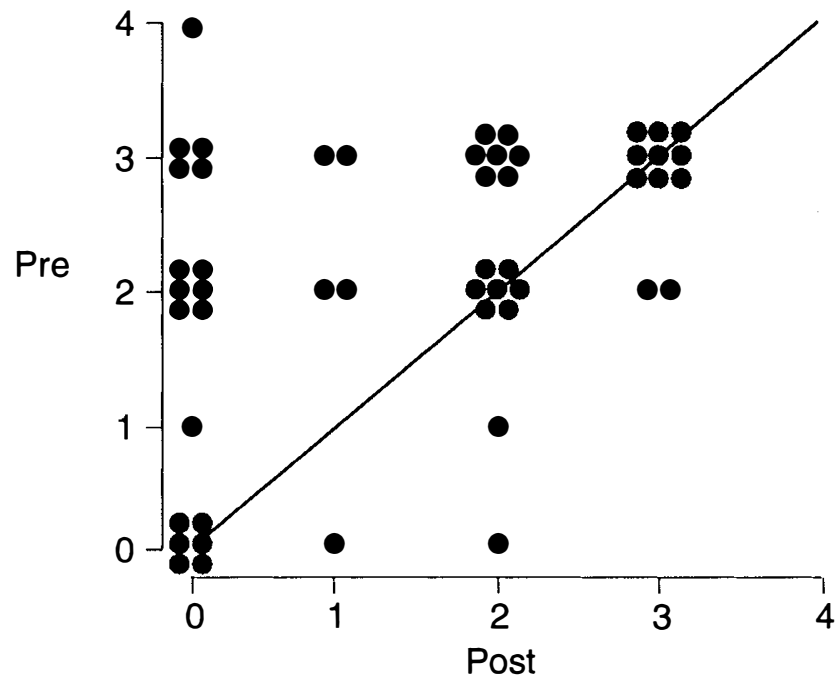

Fig. 2. Pre- and post-treatment vitreous cellular activity scores (51 injections).

stating which particular injection route had been used, although it is likely that posterior sites would have been used in most of the cases. The only study which provides exact information on the effects of single posterior peri-

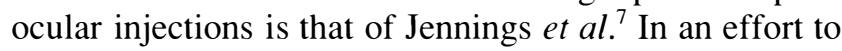
determine the mode of action of posterior sub-Tenon's steroid injections in cystoid macular oedema secondary to uveitis, they studied 12 treated eyes measuring visual acuities and blood-retinal barrier permeability (fluorescein angiography and vitreous fluorophotometry). They found that $50 \%$ of the eyes had an improvement in visual acuity which lasted for at least 4 weeks. There are no published studies of the efficacy of orbital floor steroid injections in the treatment of uveitis.

In this uncontrolled and primarily retrospective study of 33 eyes with uveitis due to a variety of causes, an improvement in visual acuity or inflammatory activity occurred after $48 \%$ of 54 orbital floor steroid injections. The duration of effect averaged 9 weeks and exceeded 4 weeks after $84 \%$ of the effective injections. The patient's age and sex, the disease aetiology, disease duration, pre-treatment visual acuity, macular or disc oedema prior to treatment, and type and dose of steroid injection had no influence on treatment outcome. It was notable that a number of patients with macular oedema and a relatively low level of inflammatory activity prior to treatment experienced an improvement in visual acuity without any change in inflammatory activity, presumably as a result of direct effects on the degree of macular oedema. Since fluorescein angiography was not carried out routinely on the eyes included in this study no more can be said about the mechanism of action of the orbital floor steroids in these eyes. Seven injections were administered to eyes with good acuity (6/9 or 6/6) for control of vitreous inflammatory activity. The response rate from these 7 injections did not differ significantly from that produced by the injections administered to eyes with reduced visual acuity, whether this was or was not associated with clinically apparent macular oedema. 
An intriguing aspect of this study is that the response to any particular injection was not predictive of the response to subsequent or even preceding injections. There is no clear explanation for this finding, although it does suggest that orbital floor steroid injections have an inherent variability of efficacy. This variability may be due to differences in techniques of administration, but this is unlikely since all the injections were administered by experienced ophthalmologists. A more reasonable explanation is that the time course of dispersion of steroids from the region of the orbital floor may vary considerably from one injection to another. In practical terms this finding indicates that a failure to achieve a response to one orbital floor steroid injection should not deter the clinician from undertaking further injections to achieve a response.

This study is limited by its primarily retrospective nature and the lack of a control group. However it does provide a useful overview of the efficacy of orbital floor steroid injections as they might be used clinically in the management of uveitis with significant posterior segment inflammation. In any retrospective study it is difficult to be sure of the precise indication for undertaking treatment in any individual case but it is a sine qua non that treatment was undertaken because it was thought clinically to be justified. This is an especially important consideration when mild disease activity does not necessarily justify treatment and may spontaneously improve. In this series the need for treatment is supported by $87 \%$ of the eyes having a pretreatment acuity of $6 / 12$ or less, and the remainder having a vitreous inflammatory score of 2 or more.

The overall positive response rate of $48 \%$ at first appears disappointing, but this needs to be put into context by comparison with other treatment modalities. For this the most relevant information relates to the treatment of pars planitis. Schlaegel et al., ${ }^{8}$ using anterior periocular methylprednisolone every 2-6 weeks, reported a rapid visual improvement (within 2 months) in 5 of 24 eyes and a slower improvement in a further 9 eyes, amounting to an overall response rate with prolonged therapy of $58 \%$. In the study by Godfrey et al. ${ }^{6}$ in which repeated periocular steroid injections produced improvement in $71 \%$ of eyes, only $40 \%$ of eyes improved on systemic steroid therapy equivalent to prednisolone $15 \mathrm{mg} /$ day or less and $60 \%$ of eyes improved on the equivalent of prednisolone $20 \mathrm{mg} /$ day or more.

It is thus clear that a $48 \%$ therapeutic response rate is a successful outcome. In support of the relative efficacy of orbital floor steroid injections in this particular patient population is that of 6 eyes that had failed to respond to systemic steroids, 5 (83\%) responded to orbital floor ster- oid injection. Whether posterior sub-Tenon's steroid injections, because of their closer proximity to the globe, would improve on the $48 \%$ response rate and the variability of response rates, and whether any such increase justifies the greater risk of globe perforation, would need to be addressed by another study.

In conclusion, orbital floor steroid injections are a useful therapeutic option in the management of posterior segment inflammation in uveitis. They are particularly applicable to patients with active disease in one eye only and when there are specific contraindications to systemic steroid therapy. There is no evidence to suggest that they are any less effective than systemic steroids in controlling disease activity. In fact animal studies indicate that periocular injections produce higher intraocular steroid levels than systemic therapy. It is interesting that in this study, failure to respond to orbital floor steroid was not predictive of a significant lack of response to subsequent injections. This obviously indicates that repeated trials of orbital floor steroid injection are justified if the clinical situation is appropriate. The major limitation to long-term management of uveitis with orbital floor steroid injections is the limited duration of action, the mean duration being 9 weeks. Whether any particular individual can be managed on a long-term basis with repeated orbital floor steroid injections will of course depend upon the patient's tolerance of periocular steroid injection, the degree of disease activity and the individual circumstances with regard to systemic steroid therapy.

Key words: Periocular injections, Steroids, Treatment, Uveitis.

\section{REFERENCES}

1. Hyndiuk RA, Reagan MG. Radioactive depot-corticosteroid penetration into monkey ocular tissue. I. Retrobulbar and systemic administration. Arch Ophthalmol 1968;80: 499-503.

2. Levine ND, Aronson SB. Orbital infusion of steroids in the rabbit. Arch Ophthalmol 1970;83:599-607.

3. Schlaegel TF. Essentials of uveitis. Boston: Little, Brown, 1969:41-3.

4. Smith RE, Nozik RA. Uveitis: a clinical approach to diagnosis and management. 2nd ed. Baltimore: Williams and Wilkins, 1989:63-8.

5. Nozik RA. Periocular injections of steroids. Trans Am Acad Ophthalmol Otolaryngol 1972;76:695-703

6. Godfrey WA, Smith RE, Kimura SJ. Chronic cyclitis: corticosteroid therapy. Trans Am Ophthalmol Soc 1976;74:178-86.

7. Jennings T, Rusin MM, Tessler HH, Cunha-Vaz JG. Posterior sub-Tenon's injections of corticosteroids in uveitis patients with cystoid macular oedema. Jpn J Ophthalmol 1988;32: 385-91.

8. Schlaegel TF, Weber JC. Treatment of pars planitis. II. Corticosteroids. Surv Ophthalmol 1977;22:120-30. 